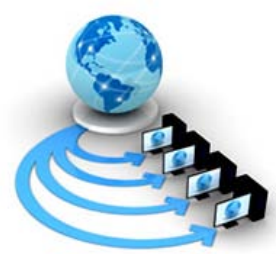

\author{
Available Online at www.ijarcs.info
}

\title{
AN EFFICIENT TECHNIQUE FOR LOSSLESS ADDRESS DATA COMPRESSION USING ADAPTIVE SPIHT ALGORITHM IN WSN
}

\author{
Sanjay Mainalli \\ Research Scholar - Bhagwant University \\ Ajmer - Rajasthan - India
}

\author{
Dr. Kalpana Sharma \\ Dept. of Computer Science \\ Bhagwant University - Ajmer - Rajasthan
}

\begin{abstract}
The computer is becoming more and more powerful day by day. Data compression is a popular approach to reducing data volumes and hence lowering disk I/O and network data transfer times. While several lossy data compression techniques have demonstrated excellent compression ratios, lossless data compression techniques are still among the most popular ones. Sensor networks represent a non-traditional source of information, as readings generated by sensors flow continuously, leading to an infinite stream of data. Sensors are non-reactive elements which are used to monitor real life phenomena, such as live weather conditions, network traffic, etc. They are usually organized into networks where their readings are transmitted using low level protocols.
\end{abstract}

Keywords : Adaptive set of SPIHT algorithm, WSN, LIS, LIP and LSP

\section{INTRODUCTION}

Wireless Sensor Networks (WSNs) are a collection of sensors and actuators which are used to monitor environmental variations at various locations [1,2]. WSN consists several nodes which are connected with other nodes for performing certain functions such sensing, exchanging data and so on. The sensor node is designed with radio transceiver, transducer, microcontroller and power supply [3]. The transducer generates electrical signals based on the environmental and physical variations. The transceiver is performed data transmission and reception function in which microcontroller is used for data processing [4]. The sensor nodes are gathered the environmental data which are communicate through radio transceiver and it is connected to the satellite network. There are various applications of WSN includes surveillance, localization, tracking and object detection [5].

In the applications of WSN, the sensor nodes are uses mostly battery-powered for long-term monitoring of environments. The energy saving of those batteries are essential for improving the lifetime of sensor network where the most energy is consumed during data transmission [6]. For this issue, data compression which reduce the amount of data is one of the best solution to save energy in WSN. Besides, the improvement in data compression algorithms has become important in multimedia WSN applications. Image compression techniques are developed to reduce the size of the image [5]1. The applications using images such as medical imaging, surveillance, remote sensing, cameras and video-on-demand systems contain huge amounts of data to transmit. Therefore, radio transceiver nodes consume more energy than receiving nodes [7]. Due to resources constraints such as processor speed and memory, there are several compression algorithms are inappropriate for real-time environment. An energy efficient compression techniques are more essential to overcome these issues [6]1.
Recently, several researchers proposed various compression techniques for data compression in wireless sensor network. The compression techniques are mainly categorized into two techniques such as lossy compression and lossless compression [8]. In the lossless compression, the redundancy is a reversible process and no information loss where in lossy compression certain portion of the information is removed. The lossy compression techniques also classified into two types such as transform-based and non-transformed based techniques [9]. In lossy compression, the transform-based techniques includes Discrete Cosine Transform (DCT) [10] and Discrete Wavelet Transform (DWT) [11] wherein non-transform includes Vector Quantization (VQ) [12]. The lossless image compression techniques includes two approaches such as de correlation and entropy coding [9]1. De correlation includes prediction based, multi-compression based and transform-based techniques and entropy coding consists Run-length coding (RLC) and variable-length coding (VLC) [13]. Moreover, a lossless address data compression based on quadtree clustering in WSN is proposed $[14,15,16]$.

An important issue in managing sensor data streams is aggregating the values produced by a subset of sources within a time interval. Here the clustering approach is done using quadtree clustering remains to produce lesser compression rate which acts as a major drawback. The possible extension here will be to include optimal clustering approach and advanced compression scheme like SPIHT algorithms for better compression rate.

\section{RESEARCH OBJECTIVES \& APPROCH}

- Aim

The major aim of the research is to develop an efficient technique for lossless address data compression using adaptive Set Partitioning in Hierarchical Trees (SPIHT). 


\section{- Objectives}

To achieve the above mentioned aim, the research has following objectives:

1. To explore the various conventional techniques and its challenges in lossless address data compression for Wireless Sensor Networks (WSNs).

2. To achieve the greater compression rate using adaptive Set Partitioning in Hierarchical Trees (SPIHT).

\section{REVIEW WORK ALREADY DONE ON THE SUBJECT}

In WSN, data compression is achieved using Joint Photographic Experts Group (JPEG) which is a popular DCT compression scheme $[17,19]$. The fundamental concept of DCT is to convert a signal into basic frequency components. For the application of compression, an image is divided into several blocks. Then, the sum of cosine functions on different frequencies can be statistically used to express each block of an image [18]. This approach is analyzed in diverse manners, including reducing the computation complexity, increasing the compression ratio, and minimizing power consumption [12]1. The power consumption of DCT-based techniques is more than DWT-based techniques [20].

Discrete Wavelet Transform (DWT) is used to overcome the weakness of DCT-based techniques. Furthermore, this is to increase the features of DCT that is frequency and localization [21], Mu [22] proposed an image compression technique based on 2Ddiscrete wavelet transform Embedded zero trees of wavelet transform (EZW). It is designed for two dimension, but it is also used in other dimensions. In EZW, encoder compresses the image into a bit stream and it is based on progressive encoding [23]. The input image decomposes into wavelet coefficients which is multi-pass process. Hamdi [24] proposed an approach based on EZW for image compression where EZW offers two types of resolution such as high and low resolution. High resolution is used for the regions where interference is detected, while low resolution is used for other regions. The results verified that the approach is better for saving power and bandwidth. Moreover, packet loss is an important factor due to the fact that EZW is vulnerable against packet losses [25].

Zhang [15]1 proposed a lightweight lossless data compression technique that balances the performance between compression and decompression. This Bit plane bitmap Quadtree (BQ-Tree) based approach encodes the bitmaps of raster bit planes as compact quadtrees that compress and index rasters simultaneously. Nelson [16]1 proposed a simple quadtree based algorithm to compress the graph without read the entire graph into the main memory. This algorithm uses the quadtree data structure that is implicitly created to produce the compressed graph output. Yang [14]1 proposed an image compression technique using quadtree decomposition to reduce bit rate and blocking effect by employing the error diffusion technique. The results shows that the proposed technique achieves better performance based on the quantitative evaluation and visual quality.

Wang \& Zhang, [26] proposed SPIHT with image stitching to make multi-view image compression for WMSN and to remove the overlap and spatial redundancy. Image sensors first captured images and sent images to microprocessor to achieve image stitching and eliminate overlap redundancy. Then stitched image was compressed with modified SPIHT coding to reduce amount of transmitted bits. Their simulation result indicated that the data to be transmitted can be reduced by $10-45 \%$ using the stitched images. EBCOT is the highest algorithm in the performance of compression quality is proposed [27]. Nevertheless, the process of Tier- 1 is responsible for arithmetic encoding and context formation for encoding code block. Therefore, consumed most of encoder's power and consequently increase power consumption, computational complexity, memory required and processing time. Thus, EBCOT not very beneficial for power constrained WMSN.

\section{CURRENT WORK AND PRELIMINARY RESULTS}

The main objective of the proposed methodology is to provide an efficient technique for lossless address data compression using adaptive Set Partitioning in Hierarchical Trees (SPIHT) in Wireless Sensor Networks (WSNs). The proposed data compression technique is applied to any block of binary address data words which fulfils the following conditions.

i) Address data words should be separate from one another and no repetition is permitted. This requirement is essential, since the addresses have to be unique to differentiate one from the other.

ii) The order of the data words is irrelevant. That is, it should be a set.

iii) The width of all the data words should be same.

Repetition or duplicate words cause uncertainty in the process of compression. Therefore, Repetition or duplicate words are not allowed. The proposed technique applies a set rather than a sequence during compression and decompression to overcome the information is lost.

Set Partitioning in Hierarchal Tree (SPIHT) is a powerful wavelet based image compression algorithm that achieved very compact output bit stream than Embedded Zero Tree of wavelet coefficients (EZW) without adding an entropy encoder. This improves its efficiency based on computational complexity. In the SPIHT, spatial orientation tree (SOT) arrangement is used to connect the coefficients after the (DWT) is applied to decompose an image into different sub bands. Furthermore, progressive mode in SPIHT permits the process of coding/decoding to be stopped at any stage of the compression. Sorting pass and the refinement passes are used in coding process in SPIHT. It consists three lists namely list of insignificant sets (LIS), list of insignificant pixels (LIP) and a list of significant pixels (LSP) are used to store coding information. Fig. 1 shows a block diagram of SPIHT scheme for data compression.

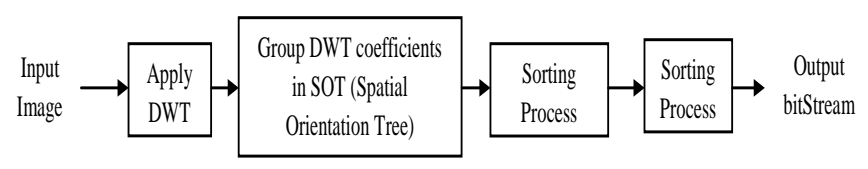

Fig.1 Proposed SPIHT scheme for Data Compression

\section{PERFORMANCE EVALUATION}

The following evaluation parameters are to be estimated for proving the superiority of the proposed SPIHT based lossless address data compression techniques. 
a) Peak Signal to Noise Ratio (PSNR)

b) Compression Ratio

c) Throughput

d) End-to-End delay

e) Execution time

\section{NEED FOR THE PROPOSED RESEARCH WORK}

The proposed research solves data compression problem in quadtree technique using an efficient technique for lossless address data compression using adaptive Set Partitioning in Hierarchical Trees (SPIHT). Due to the efficient lossless address data compression of proposed adaptive SPIHT, the transmission of data consumes less energy. Therefore, lifetime of the sensor network is to be improved. Moreover, the proposed work increases the speed of the transmission due to reduced size of the packet.

\section{BIBLIOGRAPHY}

[1] Yang, K. (2014). Wireless sensor networks. Principles, Design and Applications.

[2] Potdar, V., Sharif, A., \& Chang, E. (2009, May). Wireless sensor networks: A survey. In Advanced Information Networking and Applications Workshops, 2009. WAINA'09. International Conference on (pp. 636-641). IEEE.

[3] Akyildiz, I. F., Su, W., Sankarasubramaniam, Y., \&Cayirci, E. (2002). Wireless sensor networks:a survey. Computer networks, 38(4), 393-422.

[4] Mahmood, M. A., Seah, W. K., \& Welch, I. (2015). Reliability in wireless sensor networks: A survey and challenges ahead. Computer Networks, 79, 166-187.

[5] Marcelloni, F., \&Vecchio, M. (2009). An efficient lossless compression algorithm for tiny nodes of monitoring wireless sensor networks. The Computer Journal, 52(8), 969-987.

[6] Sheltami, T., Musaddiq, M., \&Shakshuki, E. (2016). Data compression techniques in Wireless Sensor Networks. Future Generation Computer Systems, 64, 151-162.

[7] Yang, S., Wang, S., Liu, Z., Wang, M., \& Jiao, L. (2014). Improved Bandelet with heuristic evolutionary optimization for image compression. Engineering Applications of Artificial Intelligence, 31, 27-34.

[8] ZainEldin, H., Elhosseini, M. A., \& Ali, H. A. (2015). Image compression algorithms in wireless multimedia sensor networks: A survey. Ain Shams Engineering Journal, 6(2), 481-490.

[9] Yang, M., \&Bourbakis, N. (2005, August). An overview of lossless digital image compression techniques. In Circuits and systems, 2005. 48th Midwest symposium on (pp. 1099-1102). IEEE.

[10] Saha, S. (2000). Image compression from DCT to wavelets: a review. Crossroads, 6(3), 12-21.

[11] Chew, L. W., Ang, L. M., \& Seng, K. P. (2008, August). Survey of image compression algorithms in wireless sensor networks. In Information Technology, 2008. ITSim 2008. International Symposium on (Vol. 4, pp. 1-9). IEEE.

[12] Mammeri, A., Hadjou, B., \&Khoumsi, A. (2012). A survey of image compression algorithms for visual sensor networks. ISRN Sensor Networks, 2012.
[13] Lv, J., Li, S., \& Zhang, X. (2017). A novel auxiliary data construction scheme for reversible data hiding in JPEG images. Multimedia Tools and Applications, 1-13.

[14] Yang, F. J., Lien, C. Y., Chen, P. Y., \& Hsu, C. L. (2016, March). An efficient quadtree-based block truncation coding for digital image compression. In Advanced Information Networking and Applications Workshops (WAINA), 2016 30th International Conference on (pp. 939-942). IEEE.

[15] Zhang, J., You, S., \&Gruenwald, L. (2015, October). Quadtree-based lightweight data compression for largescale geospatial rasters on multi-core CPUs. In Big Data (Big Data), 2015 IEEE International Conference on (pp. 478-484). IEEE.

[16] Nelson, M., Radhakrishnan, S., Chatterjee, A., \&Sekharan, C. N. (2015, October). On compressing massive streaming graphs with Quadtrees. In Big Data (Big Data), 2015 IEEE International Conference on (pp. 2409-2417). IEEE.

[17] Wallace, G. K. (1992). The JPEG still picture compression standard. IEEE transactions on consumer electronics, 38(1), xviii-xxxiv.

[18] Jeong, H., Kim, J., \& Cho, W. K. (2004). Low-power multiplier less DCT architecture using image correlation. IEEE Transactions on Consumer Electronics, 50(1), 262-267.

[19] Zheng, W., \& Liu, Y. (2011, April). Research in a fast DCT algorithm based on JPEG. In Consumer Electronics, Communications and Networks (CECNet), 2011 International Conference on (pp. 551-553). IEEE.

[20] Pham, D. M., \& Aziz, S. M. (2013, April). An energy efficient image compression scheme for Wireless Sensor Networks. In Intelligent Sensors, Sensor Networks and Information Processing, 2013 IEEE Eighth International Conference on(pp. 260-264). IEEE.

[21] Mallat, S. G. (1989). A theory for multi resolution signal decomposition: the wavelet representation. IEEE transactions on pattern analysis and machine intelligence, 11(7), 674-693.

[22] Mu, Y., Murali, B., \& Ali, A. L. (2005, October). Embedded image coding using zero trees of wavelet coefficients for visible human dataset. In Signals, Systems and Computers, 2005. Conference Record of the Thirty-Ninth Asilomar Conference on (pp. 276280). IEEE.

[23] Shingate, V. S., Sontakke, T. R., \& Talbar, S. N. (2010). Still image compression using embedded zerotree wavelet encoding. International Journal of Computer Science \& Communication, 1(1), 21-24.

[24] Hamdi, M., Boudriga, N., \& Obaidat, M. S. (2008). Bandwidth-effective design of a satellite-based hybrid wireless sensor network for mobile target detection and tracking. IEEE Systems Journal, 2(1), 74-82.

[25] Chithra, P. L., \& Thangavel, P. (2010, November). A fast and efficient memory image codec (encoding/decoding) based on all level curve let transform co-efficients with SPIHT and Run Length Encoding. In Recent Advances in Space Technology Services and Climate Change (RSTSCC), 2010 (pp. 174-178). IEEE.

[26] Wang, J., \& Zhang, F. (2010, June). Study of the Image Compression based on SPIHT Algorithm. In Intelligent 
Sanjay Mainalli et al, International Journal of Advanced Research in Computer Science, 9 (1), Jan-Feb 2018,574-577

computing and cognitive informatics (ICICCI), 2010 international conference on (pp. 130-133). IEEE.
[27] Taubman, D. (2000). High performance scalable image compression with EBCOT. IEEE Transactions on image processing, 9(7), 1158-1170. 